\title{
Elevated B-type natriuretic peptide (BNP) and soluble thrombomodulin (sTM) indicates severity and poor prognosis of sepsis
}

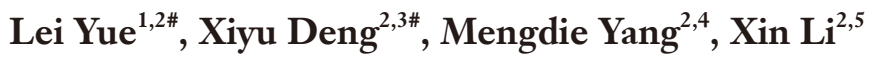 \\ ${ }^{1}$ Department of Medical Education, Sichuan Provincial People's Hospital, University of Electronic Science and Technology of China, Chengdu, \\ China; ${ }^{2}$ Chinese Academy of Sciences Sichuan Translational Medicine Research Hospital, Chengdu, China; ${ }^{3}$ Department of Digestive Internal \\ Medicine, Sichuan Provincial People's Hospital, University of Electronic Science and Technology of China, Chengdu, China; ${ }^{4}$ Department of \\ Gynaecology, Sichuan Provincial People's Hospital, University of Electronic Science and Technology of China, Chengdu, China; ${ }^{5}$ Department of \\ Emergency, Sichuan Provincial People's Hospital, University of Electronic Science and Technology of China, Chengdu, China \\ Contributions: (I) Conception and design: L Yue, X Deng; (II) Administrative support: M Yang, X Li; (III) Provision of study materials or patients: M \\ Yang, X Li; (IV) Collection and assembly of data: L Yue, X Deng; (V) Data analysis and interpretation: L Yue, X Deng; (VI) Manuscript writing: All \\ authors; (VII) Final approval of manuscript: All authors. \\ \#These authors contributed equally to this work. \\ Correspondence to: Mengdie Yang. Department of Gynaecology, 585 Honghe Dadao, Longquanyi District, Chengdu, China. \\ Email: mengdie2021222@163.com; Xin Li. Department of Emergency, West Road Section 2, First Ring Road, Chengdu, China, \\ Email: lixin20211008@163.com.
}

Background: To study the predictive value of B-type natriuretic peptide (BNP) and soluble thrombomodulin (sTM) in the severity stratification and prognosis evaluation of sepsis.

Methods: The clinical data of 137 sepsis patients diagnosed and treated in Sichuan Provincial People's Hospital from May 2018 to November 2020 were retrospectively analyzed. Meanwhile, 121 healthy individuals were selected as the control group. Patients with sepsis were allocated into the mild group, severe group, and shock group according to the severity. According to the 28-day prognosis, the patients were allocated into the death group and survival group. The plasma BNP and serum sTM levels in different groups were compared, and their prognostic value was evaluated.

Results: Patients with sepsis had significantly higher levels of BNP and sTM than the healthy control group $(\mathrm{P}<0.05)$. The levels of BNP and sTM in the mild group were significantly lower than those in the severe group and shock group, and both BNP and sTM were positively correlated with Acute Physiology and Chronic Health Status (APACHE) II score ( $\mathrm{r}=0.595,0.516, \mathrm{P}<0.05)$. The levels of BNP and sTM in the death group were significantly higher than those in the survival group $(\mathrm{P}<0.05)$. The area under curve (AUC) of BNP combined with sTM was significantly greater than that of BNP or sTM alone for the prognosis of sepsis $(\mathrm{P}<0.05)$. When the cut-off value of BNP was $625.68 \mathrm{pg} / \mathrm{mL}$, the sensitivity and specificity were $77.42 \%$ and $89.42 \%$, respectively. When the cut-off value of sTM was $10.53 \mathrm{ng} / \mathrm{mL}$, the sensitivity and specificity were $83.87 \%$ and $94.34 \%$, respectively.

Conclusions: Patients with sepsis have significantly higher serum BNP and sTM levels which are positively correlated with the severity of the disease. Both of the 2 indexes have good prognostic value, and the predictive value is higher when combined.

Keywords: B-type natriuretic peptide (BNP); soluble thrombomodulin (sTM); sepsis; severity; prognosis

Submitted Mar 18, 2021. Accepted for publication May 13, 2021.

doi: 10.21037/apm-21-1048

View this article at: http://dx.doi.org/10.21037/apm-21-1048 


\section{Introduction}

Sepsis is the systemic inflammatory response syndrome of the body caused by infection, predominantly bacterial infection. In severe cases, it can lead to deadly dysfunction of organs or circulation. It is a common complication of specific conditions such as burns, infections, and surgical procedures $(1,2)$. According to current clinical practice, septic patients are divided into 3 subtypes: sepsis, severe sepsis, and septic shock, according to their organ involvement and hemodynamics. Severe sepsis and septic shock are important causes of death for intensive care unit (ICU) patients (3). The Acute Physiology and Chronic Health Status (APACHE) II scoring system, as well as hemodynamic monitoring, are used to grade the patient's condition and help predict prognosis. However, these indicators are not perfect references for clinical diagnosis and treatment, as there are too many evaluation parameters, which makes the evaluation untimely. Additionally, it is invasive (4). Therefore, doctors have been committed to finding an ideal non-invasive indicator for sepsis. The pathology of sepsis mainly consists of uncontrolled inflammation, dysfunction of coagulation, and microcirculation dysfunction caused by vascular endothelial damage. Based on the pathology of sepsis, a variety of studies have shown that some biomarkers are good indicators for diagnosis and prognosis, which aids in developing a more comprehensive understanding of the disease in specific patients (5). B-type natriuretic peptide (BNP) and soluble thrombomodulin (sTM) are released out of endothelial cells upon damage. Studies have confirmed that the levels of BNP and sTM in patients with sepsis significantly increase $(6,7)$. However, the clinical significance of changes in BNP and sTM levels in septic patients is still unclear. This study explores the diagnostic and prognostic value of BNP and sTM in sepsis.

We present the following article in accordance with the STARD reporting checklist (available at http://dx.doi. org/10.21037/apm-21-1048).

\section{Methods}

\section{Patients}

The clinical data of 137 septic patients (sepsis group) diagnosed and treated in Sichuan Provincial People's Hospital from May 2018 to November 2020 were retrospectively analyzed. The inclusion criteria were as follows: diagnosed with sepsis (8); tested BNP and sTM levels within 24 hours of admission; the clinical data of the patients was complete. The exclusion criteria were as follows: Recent history of immunotherapy; patients with diseases of the blood system or immune system; patients with acute and chronic thromboembolic diseases; patients with liver, kidney, and lung diseases. In addition, 121 healthy people who came to our hospital for physical examination during the same period were selected as the control group. The general data of the sepsis group and the control group are shown in Table 1, which were statistically significant $(\mathrm{P}>0.05)$.

To explore the prognostic value of BNP and sTM levels in septic patients, the septic patients were divided into the mild group, severe group, and shock group according to sepsis classification standards (9). The patients were allocated into the death group and survival group according to their 28-day prognosis.

The study followed the Declaration of Helsinki (as revised in 2013) and was approved by the ethics committee of Sichuan Provincial People's Hospital. Approval number was waived as this is a retrospective research. All patient who conformed to criteria were involved. Written consent was obtained from all patients.

\section{Determination of BNP and sTM levels and APACHE II score}

The general information of the enrolled patients was collected, mainly including gender, age, vital signs, and blood routine. In the sepsis group, venous blood was collected within 24 hours after admission. In the control group, venous blood was collected on the day of the physical examination. The blood was kept at room temperature for 2 hours, and centrifuged at a speed of $1,000 \mathrm{r} / \mathrm{min}$ for approximately 20 minutes to collect serum. A fluorescence immunoassay was used to detect plasma BNP level, and an enzyme-linked immunosorbent assay was used to detect serum sTM level according to the manufacturers' instructions. Furthermore, the APACHE II score was evaluated on the 1st day after admission of the septic patient.

\section{Statistics}

SPSS 20.0 software was used for statistical processing. All data was subjected to normal analysis and homogeneity of variance tests. All data were expressed as mean \pm standard deviation. The comparison between groups was performed 
Table 1 General information of the patients

\begin{tabular}{lccccc}
\hline Groups & Cases & Gender & Age & Body mass index $\left(\mathrm{kg} / \mathrm{m}^{2}\right)$ & APACHE II score \\
\hline Sepsis & 137 & $71 / 66$ & $45.24 \pm 11.64$ & $24.15 \pm 2.82$ & $13.09 \pm 3.42$ \\
Control & 121 & $62 / 59$ & $47.19 \pm 10.35$ & $23.64 \pm 2.52$ & - \\
$t / \chi^{2}$ & & 0.009 & 1.414 & 1.523 & - \\
$P$ & & 0.925 & 0.159 & 0.129 & - \\
\hline
\end{tabular}

APACHE, Acute Physiology and Chronic Health Status.

Table 2 Comparison of BNP and sTM levels between the sepsis group and the control group (mean SD)

\begin{tabular}{lccc}
\hline Group & Cases & BNP $(\mathrm{pg} / \mathrm{mL})$ & $\mathrm{sTM}(\mathrm{ng} / \mathrm{mL})$ \\
\hline Sepsis & 137 & $383.49 \pm 130.24$ & $9.27 \pm 3.19$ \\
Control & 121 & $56.87 \pm 22.76$ & $3.87 \pm 1.21$ \\
$t$ & & 27.216 & 17.537 \\
P & & $<0.001$ & $<0.001$ \\
\hline
\end{tabular}

BNP, B-type natriuretic peptide; sTM, soluble thrombomodulin.

by a $t$ test and a one-way analysis of variance. Spearman correlation analysis was used for the correlations between BNP and sTM levels and disease severity, and Pearson correlation analysis was used for the correlation with prognosis. The predictive value of BNP and sTM levels on the prognosis of sepsis patients was analyzed by an ROC curve. The area under the ROC curve (AUC) is used to reflect the predictive power. $Z$ test was used in the comparison of the AUC values. $\mathrm{P}<0.05$ indicated that the difference was statistically significant.

\section{Results}

\section{Comparison of BNP and sTM levels between septic patients and bealthy controls}

The levels of BNP and sTM in patients with sepsis were significantly higher than those in the control group (Table 2, $\mathrm{P}<0.05)$.

\section{Comparison of BNP and sTM levels in patients with different conditions}

The levels of BNP and sTM in the mild group were significantly lower than those in the severe and shock groups, and both BNP and sTM were positively correlated with APACHE II score $(\mathrm{r}=0.595,0.516 ; \mathrm{P}<0.05)$ (Table 3 and Figures 1 and 2).
Comparison of BNP, sTM levels, and APACHE II scores in patients with different prognoses

The levels of BNP and sTM in the death group were significantly higher than those in the survival group, and the difference was statistically significant (Table $4, \mathrm{P}<0.05$ ).

\section{The predictive value of BNP and STM for the prognosis of patients with sepsis}

The AUC of BNP combined with sTM was more significant in assessing the prognosis of sepsis than that of BNP or sTM alone $(\mathrm{P}<0.05)$. When the cut-off value of BNP was $625.68 \mathrm{pg} / \mathrm{mL}$, the sensitivity was $77.42 \%$ and the specificity was $89.42 \%$. When the cut-off value of sTM was $10.53 \mathrm{ng} / \mathrm{mL}$, the sensitivity was $83.87 \%$ and the specificity was $94.34 \%$ (Table 5 and Figure 3).

\section{Discussion}

Over the past 2 decades, the incidence of sepsis has doubled and it is now the tenth leading cause of death (10). However, the progression of the disease can be controlled to a certain extent through the active use of antibacterial drugs and advanced life-supporting treatments. Sepsis is the most common cause of death in the ICU, especially for patients with severe sepsis and septic shock. More and more clinicians have realized that in addition to standardized 
Table 3 Comparison of BNP and sTM levels among patients with different conditions (mean \pm SD)

\begin{tabular}{lcccr}
\hline Group & Cases & BNP $(\mathrm{pg} / \mathrm{mL})$ & sTM $(\mathrm{ng} / \mathrm{mL})$ & APACHE II score \\
\hline Mild & 61 & $213.64 \pm 82.41$ & $6.82 \pm 2.54$ & $9.56 \pm 2.63$ \\
Severe & 47 & $431.79 \pm 156.48^{*}$ & $9.76 \pm 3.16^{*}$ & $13.56 \pm 4.13^{\star}$ \\
Shock & 29 & $662.47 \pm 187.73^{\star \#}$ & $13.65 \pm 4.28^{* \#}$ & $19.75 \pm 3.82^{\star \#}$ \\
F & & 109.685 & 46.025 & 85.689 \\
P & & $<0.001$ & $<0.001$ & $<0.001$ \\
\hline
\end{tabular}

Compared with the severe group, ${ }^{*} \mathrm{P}<0.05$; compared with the shock group, ${ }^{\#} \mathrm{P}<0.05$. BNP, B-type natriuretic peptide; sTM, soluble thrombomodulin; APACHE, Acute Physiology and Chronic Health Status.

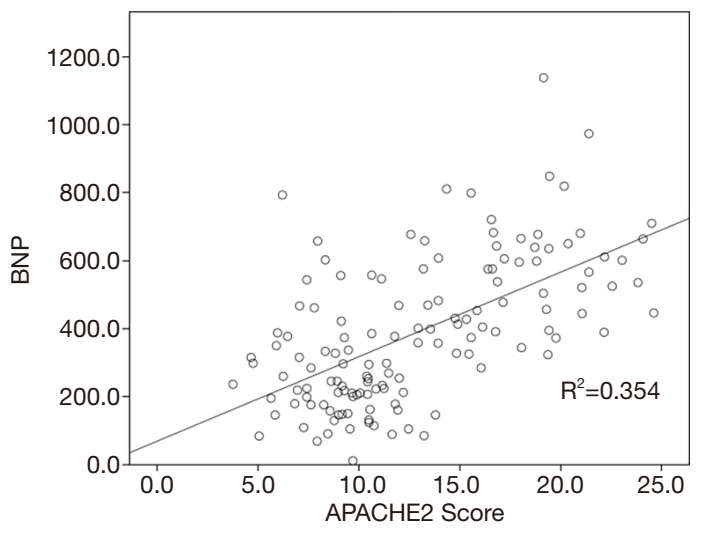

Figure 1 The correlation between BNP and APACHE II score. BNP, B-type natriuretic peptide; APACHE, Acute Physiology and Chronic Health Status.

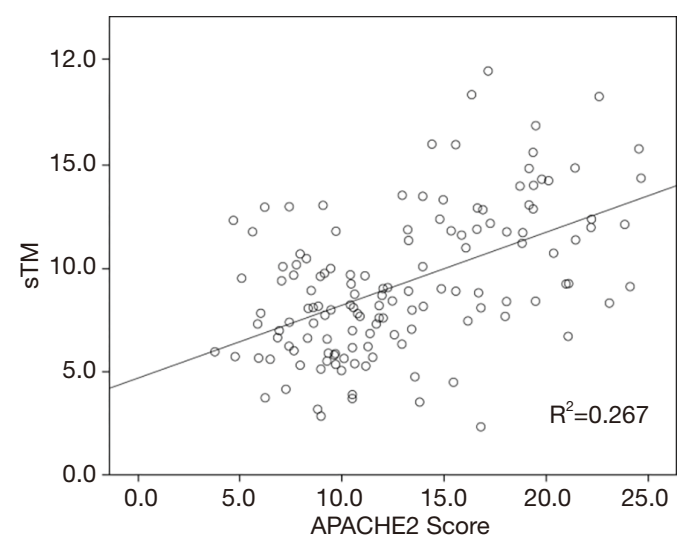

Figure 2 The correlation between sTM and APACHE II score. sTM, soluble thrombomodulin; APACHE, Acute Physiology and Chronic Health Status.
Table 4 Comparison of BNP and sTM levels and APACHE II scores between patients with different prognoses (mean $\pm \mathrm{SD}$ )

\begin{tabular}{lccc}
\hline Group & Cases & BNP $(\mathrm{pg} / \mathrm{mL})$ & $\mathrm{sTM}(\mathrm{ng} / \mathrm{mL})$ \\
\hline Death & 31 & $872.68 \pm 160.32$ & $15.29 \pm 4.05$ \\
Survival & 106 & $240.43 \pm 121.44$ & $7.51 \pm 2.94$ \\
$t$ & & 23.622 & 11.833 \\
P & & $<0.001$ & $<0.001$ \\
\hline
\end{tabular}

BNP, B-type natriuretic peptide; sTM, soluble thrombomodulin; APACHE, Acute Physiology and Chronic Health Status.

treatment and updated diagnosis and treatment, the exact evaluation and monitoring of patients' conditions have an important role in controlling patient complications and improving overall prognosis. At present, clinical indicators for the assessment of sepsis mainly include inflammatory indicators, severity score scales, and invasive and noninvasive monitoring. However, the above methods still have certain limitations, such as complications caused by invasive monitoring, deviations in laboratory indicators in different populations and diseases, and mutual interference between various indicators. Therefore, it is urgent to find more reliable indicators to accurately evaluate the condition and improve the prognosis of patients.

$\mathrm{BNP}$ is one of the members of the natriuretic peptide family. An increase in BNP level is mainly caused by the stretch of the ventricular muscle caused by the increase in heart volume load. It is an important criterion reflecting the severity of heart failure (11). In recent years, some studies have demonstrated that $50 \%$ of sepsis patients show myocardial involvement within 48 hours of admission, and 
Table 5 The prognostic value of BNP and sTM in patients with sepsis

\begin{tabular}{lcccc}
\hline Variant & AUC & Standard error of mean & P & 95\% confidence interval \\
\hline BNP & 0.874 & 0.042 & 0.000 & $0.794-0.957$ \\
STM & 0.913 & 0.041 & 0.000 & $0.832-0.994$ \\
Combined & 0.937 & 0.034 & 0.000 & $0.865-1.000$ \\
\hline
\end{tabular}

BNP, B-type natriuretic peptide; sTM, soluble thrombomodulin.

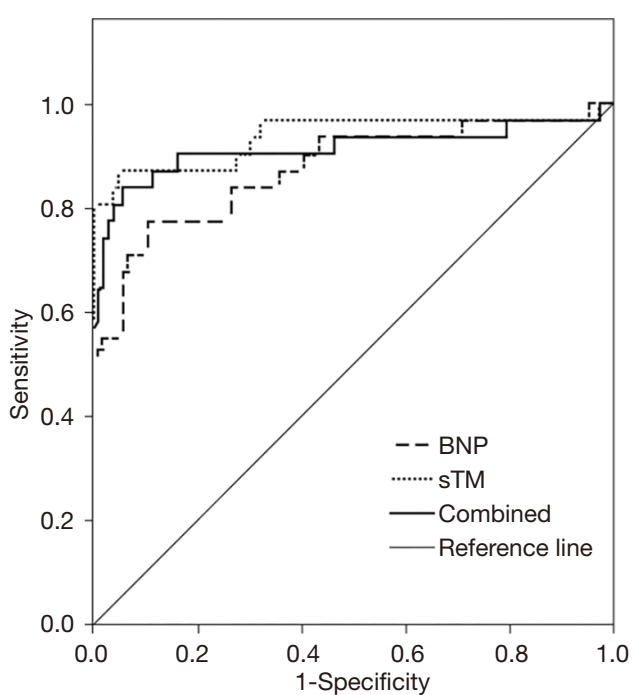

Figure 3 The prognostic value of BNP and sTM in patients with sepsis. BNP, B-type natriuretic peptide; sTM, soluble thrombomodulin.

it is believed that the cause may be related to the activation of inflammatory factors such as tumor necrosis factor- $\alpha$ (12). The emergence of myocardial depression promotes the disease progression of patients with sepsis and affects the prognosis. At present, researchers have reported that serum BNP in the blood of patients with sepsis increases, and it has a good predictive value for the prognosis of patients with septic shock (13). The results of this study also showed that the BNP level of patients with sepsis was significantly higher than that of the control group, indicating that the occurrence of sepsis is closely related to the abnormal increase in BNP level, which is consistent with the results of previous studies.

Endothelial injury is an important feature of patients with sepsis. Fani et al. (14) and others have shown that the occurrence of complications such as kidney injury in patients with sepsis is closely related to the microvascular dysfunction caused by endothelial cell injury. Additionally,
sTM is mainly secreted by vascular endothelial cells, and when endothelial damage occurs, sTM is released into the blood, leading to a dramatic increase in serum sTM. Studies by many researchers have shown that serum sTM level can be used as a marker of vascular endothelial injury, and STM has been approved in treating septic disseminated intravascular coagulation $(15,16)$. Some researchers have also found that the serum sTM level of patients with sepsis is significantly higher than that of healthy people (17). This was also seen in this study, indicating that the serum sTM level is also closely related to sepsis.

The APACHE II score is currently a more scientific, objective, and credible assessment method to assess the severity of the disease, and it has become a common assessment standard for the severity of clinical patients. In this study, the patients were grouped and analyzed according to the severity of the disease. The results showed that the levels of BNP and sTM in mild patients were significantly lower than those in the severe group and shock group, and BNP and sTM were positively correlated with the APACHE II score, suggesting that serum BNP and sTM levels are associated with sepsis. The progression of the disease is closely related and can be used as an important indicator to evaluate the development of the patient's condition. The more severe the sepsis, the stronger the possibility that the organs are involved in the uncontrolled immune and inflammatory responses, leading to disorders of myocardial microcirculation. Other pathological changes, including the reduction of myocardial contractility, and the increase in heart volume load and pressure load, are possibly responsible for the increase in serum BNP levels (18). Rodrigues et al. (19) and others believe that sTM is the initiating factor of vascular endothelial cell damage, and vascular endothelial cell damage and microcirculation disorder can promote the progression of sepsis, which forms positive feedback. The increase in sTM level will also affect humoral immune function, promote the spread of infection to multiple organs and tissues, and aggravate the disease. In addition, from the comparison between the survival group 
and the death group, it was found that the levels of BNP and STM in the death group were significantly higher than those in the survival group, suggesting that serum BNP and sTM levels are closely related to prognosis. The ROC curve was used to further analyze the prognostic value of serum BNP and sTM levels. The results showed that the AUC of BNP combined with sTM to assess the prognosis of sepsis was significantly greater than that of BNP or sTM alone. When the cut-off value of BNP was $625.68 \mathrm{pg} / \mathrm{mL}$, the sensitivity was $77.42 \%$ and the specificity was $89.42 \%$, and when the cut-off value of sTM was $10.53 \mathrm{ng} / \mathrm{mL}$, the sensitivity was $83.87 \%$ and the specificity was $94.34 \%$. These results suggested that an abnormal increase in serum BNP and sTM levels could better predict prognosis. Vallabhajosyula et al. (20) also showed that patients with severe sepsis have higher serum BNP levels, and when the result is greater than $622 \mathrm{pg} / \mathrm{mL}$, the fatality rate increases significantly. Compared with patients in the survival group, the patients in the death group had more severe disease, a higher incidence of organ dysfunction, and worse prognosis. The myocardium is inhibited by multiple factors, and the damage to the vascular endothelium is aggravated, leading to the release of a large amount of BNP and sTM. However, sepsis patients with poor prognosis also have renal function involvement, resulting in a relative decrease in the metabolism of BNP and sTM. As the clearance is reduced, generation is increased, and the values of BNP and sTM detected in the blood are higher.

In summary, serum BNP and sTM levels in patients with sepsis are significantly increased, and are positively correlated with the severity of the disease. Both BNP and sTM have good predictive value for prognosis of sepsis, and the predictive value is higher when combined BNP and sTM. The limitation of this study is that it is a singlecenter study. The source of the selected samples was mainly patients admitted to our hospital, resulting in an insufficient number of selected cases. When subgroup analysis is involved, the sample size is small, so it is necessary to accumulate multi-center and large-scale data for further analysis.

\section{Acknowledgments}

Funding: None.

\section{Footnote}

Reporting Checklist: The authors have completed the STARD reporting checklist. Available at http://dx.doi.org/10.21037/ apm-21-1048

Data Sharing Statement: Available at http://dx.doi. org/10.21037/apm-21-1048

Conflicts of Interest: All authors have completed the ICMJE uniform disclosure form (available at http://dx.doi. org/10.21037/apm-21-1048). The authors have no conflicts of interest to declare.

Ethical Statement: The authors are accountable for all aspects of the work in ensuring that questions related to the accuracy or integrity of any part of the work are appropriately investigated and resolved. This study was approved by the ethics committee of Sichuan Provincial People's Hospital. Approval number was waived as this is a retrospective research. All procedures performed in this study involving human participants were in accordance with the Declaration of Helsinki (as revised in 2013). Written consent was obtained from all patients.

Open Access Statement: This is an Open Access article distributed in accordance with the Creative Commons Attribution-NonCommercial-NoDerivs 4.0 International License (CC BY-NC-ND 4.0), which permits the noncommercial replication and distribution of the article with the strict proviso that no changes or edits are made and the original work is properly cited (including links to both the formal publication through the relevant DOI and the license). See: https://creativecommons.org/licenses/by-nc-nd/4.0/.

\section{References}

1. Font MD, Thyagarajan B, Khanna AK. Sepsis and Septic Shock - Basics of diagnosis, pathophysiology and clinical decision making. Med Clin North Am 2020;104:573-85.

2. Fleischmann C, Scherag A, Adhikari NK, et al. Assessment of Global Incidence and Mortality of Hospital-treated Sepsis. Current Estimates and Limitations. Am J Respir Crit Care Med 2016;193:259-72.

3. Shankar-Hari M, Harrison DA, Rubenfeld GD, et al. Epidemiology of sepsis and septic shock in critical care units: comparison between sepsis-2 and sepsis-3 populations using a national critical care database. Br J Anaesth 2017;119:626-36.

4. Huda AQ, Karim MR, Mahmud MA, et al. Use of Acute Physiology and Chronic Health Evaluation (APACHE)-II 
and Red Cell Distribution Width (RDW) for Assessment of Mortality of Patients with Sepsis in ICU. Mymensingh Med J 2017;26:585-91.

5. Chen J, He Y, Zhou L, et al. Long non-coding RNA MALAT1 serves as an independent predictive biomarker for the diagnosis, severity and prognosis of patients with sepsis. Mol Med Rep 2020;21:1365-73.

6. Li H, Shan-Shan Z, Jian-Qiang K, et al. Predictive value of C-reactive protein and NT-pro-BNP levels in sepsis patients older than 75 years: a prospective, observational study. Aging Clin Exp Res 2020;32:389-97.

7. Katayama S, Nunomiya S, Koyama K, et al. Markers of acute kidney injury in patients with sepsis: the role of soluble thrombomodulin. Crit Care 2017;21:229.

8. Scherag A, Schöneweck F, Kesselmeier M, et al. Genetic Factors of the Disease Course after Sepsis: A GenomeWide Study for 28Day Mortality. EBioMedicine 2016;12:239-46.

9. Goldstein B, Giroir B, Randolph A. International pediatric sepsis consensus conference: definitions for sepsis and organ dysfunction in pediatrics. Pediatr Crit Care Med 2005;6:2-8.

10. Kempker JA, Martin GS. The Changing Epidemiology and Definitions of Sepsis. Clin Chest Med 2016;37:165-79.

11. Kuwahara K, Nakagawa Y, Nishikimi T. Cutting Edge of Brain Natriuretic Peptide (BNP) Research - The Diversity of BNP Immunoreactivity and Its Clinical Relevance. Circ J 2018;82:2455-61.

12. Lorente-Sorolla C, Garcia-Gomez A, Català-Moll F, et al. Inflammatory cytokines and organ dysfunction associate with the aberrant DNA methylome of monocytes in sepsis. Genome Med 2019;11:66.

Cite this article as: Yue L, Deng X, Yang M, Li X. Elevated B-type natriuretic peptide (BNP) and soluble thrombomodulin (sTM) indicates severity and poor prognosis of sepsis. Ann Palliat Med 2021;10(5):5561-5567. doi: 10.21037/apm-21-1048
13. Custodero C, Wu Q, Ghita GL, et al. Prognostic value of NT-proBNP levels in the acute phase of sepsis on lower long-term physical function and muscle strength in sepsis survivors. Crit Care 2019;23:230.

14. Fani F, Regolisti G, Delsante M, et al. Recent advances in the pathogenetic mechanisms of sepsis-associated acute kidney injury. J Nephrol 2018;31:351-9.

15. Suzuki A, Taniguchi H, Kondoh Y, et al. Soluble thrombomodulin in bronchoalveolar lavage fluid is an independent predictor of severe drug-induced lung injury. Respirology 2017;22:744-9.

16. Kawamoto E, Nago N, Okamoto T, et al. Anti-adhesive effects of human soluble thrombomodulin and its domains. Biochem Biophys Res Commun 2019;511:312-7.

17. Katayama S, Koyama K, Shima J, et al. Thrombomodulin, Plasminogen Activator Inhibitor-1 and Protein C Levels, and Organ Dysfunction in Sepsis. Crit Care Explor 2019; 1:e0013.

18. Kakoullis L, Giannopoulou E, Papachristodoulou E, et al. The utility of brain natriuretic peptides in septic shock as markers for mortality and cardiac dysfunction: A systematic review. Int J Clin Pract 2019;73:e13374.

19. Rodrigues AT, Rodrigues JT, Rodrigues CT, et al. Association between thrombomodulin and high mobility group box 1 in sepsis patients. World J Crit Care Med 2020;9:63-73.

20. Vallabhajosyula S, Wang Z, Murad MH, et al. Natriuretic Peptides to Predict Short-Term Mortality in Patients With Sepsis: A Systematic Review and Meta-analysis. Mayo Clin Proc Innov Qual Outcomes 2020;4:50-64.

(English Language Editor: C. Betlazar-Maseh) 